\title{
DÜBLIN
}

Technological University Dublin

ARROW@TU Dublin

\section{Towards Circularly Polarized Reconfigurable Huygens Source}

\author{
Adam Narbudowicz \\ Technological University Dublin, adam.narbudowicz@mydit.ie \\ Max Ammann \\ Technological University Dublin, max.ammann@tudublin.ie \\ Dirk Heberling \\ RWTH Aachen University
}

Follow this and additional works at: https://arrow.tudublin.ie/engscheleart

Part of the Electromagnetics and Photonics Commons

\section{Recommended Citation}

A. Narbudowicz, M. J. Ammann, and D. Heberling, (2015) Towards Circularly Polarized Reconfigurable Huygens Source, IEEE AP-S International Symposium on Antennas and Propagation, Vancouver, Canada, Institute of Electronic \& Electrical Engineers, pp. WE-UB.1A.9: Metamaterial Lenses and Lens Antennas, $3229,19 / 07 / 2015$.

This Conference Paper is brought to you for free and open access by the School of Electrical and Electronic Engineering at ARROW@TU Dublin. It has been accepted for inclusion in Conference papers by an authorized administrator of ARROW@TU Dublin. For more information, please contact arrow.admin@tudublin.ie, aisling.coyne@tudublin.ie,gerard.connolly@tudublin.ie. Funder: Irish Research Council

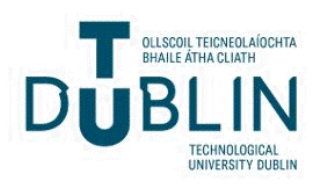




\section{Towards Circularly Polarized Reconfigurable Huygens Source}

\author{
Adam Narbudowicz ${ }^{1,2}$ \\ ${ }^{1}$ Institute of High Frequency Technology \\ RWTH Aachen University \\ Aachen, NRW, Germany \\ heberling@ihf.rwth-aachen.de
}

\author{
Max Ammann ${ }^{2}$ \\ Dirk Heberling ${ }^{1}$
${ }^{2}$ Antenna \& High Frequency Research Centre Dublin Institute of Technology
Dublin, Ireland
\{adam.narbudowicz; max.ammann\}@dit.ie

\begin{abstract}
The paper investigates an array of two reconfigurable circularly-polarized antennas with omnidirectional radiation pattern. The results are based on a previously reported antenna, which offers a steerable dipolelike radiation pattern. Its capabilities allow reception of a signal from practically any direction with steering realized by rotation of the pattern around a single axis. In this paper we propose to extend this functionality by forming an array of two such antennas oriented as a Huygens source. Simulations demonstrate six distinctive radiation patterns, each illuminating a different sector of the surrounding sphere. This allows increased antenna gain and simplification of the steering algorithm for future applications. The proposed antenna can be implemented for both MIMO and beamsteering.
\end{abstract}

\section{INTRODUCTION}

Recently, a reconfigurable antenna which offers a circularly-polarized dipole-like (i.e. torus shaped) radiation pattern was introduced [1]. With proper steering these antennas offer the reception of signals from any arbitrary angle. However the steering of the radiation pattern in [1] allows only one degree of freedom, i.e. rotation along a single axis.

In this paper we investigate an array of two such antennas. This allows additional degrees of freedom in shaping the radiation pattern. The antennas are separated by a distance of $\lambda / 2$ and are oriented in orthogonal planes (see Fig. 1). This allows the array to behave more like a Huygens source, than a linear array, i.e. it allows a unidirectional radiation pattern.

For linearly polarized antennas, Huygens sources usually combine two basic antenna shapes: a monopole/dipole and a loop. With proper spatial alignment, a unidirectional beam with up to $4.8 \mathrm{dBi}$ gain can be produced, while using simple antenna shapes [2-4]. This comes from the fact that although a monopole/dipole and a loop produce almost the same pattern shape, their polarization is orthogonal.

It is demonstrated that for CP antennas with torus-shaped radiation patterns a Huygens source can be obtained using two antennas of the same type, when they are oriented at $90^{\circ}$ with respect to each other and a phase shift of $\pm 90^{\circ}$ is applied between them [4]. Due to the current size limitation of [1], the reconfigurable antennas were spaced at $\lambda / 2$. This is considered too big for Huygens sources and therefore produced undesired sidelobes. Nevertheless, this problem is expected to vanish with future development of more compact designs.

Although the proposed work is larger and more bulky than the CP antennas presented in [3-4], it offers a much greater degree of pattern reconfiguration.

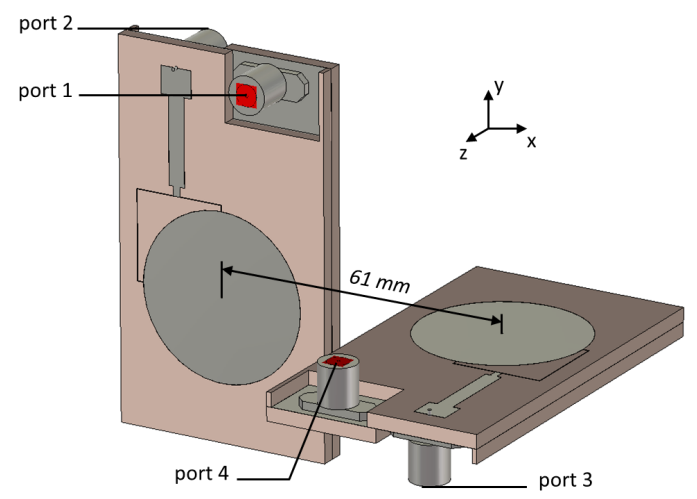

Fig. 1. Antenna orientation for the investigated array. For antenna layout and detailed description, please see [1].

\section{HUYGENS SOURCE}

The classical concept of a Huygens source relies on interference between two orthogonally-oriented torus-shaped radiation patterns. These patterns have their omnidirectional radiation cuts intersecting at two antipodal points. If the polarization is properly aligned, a constructive interference is produced at one point and a negative interference on the other, hence producing a unidirectional beam.

The reconfigurable antenna presented in [1] is perfectly suited to this purpose, as it produces a torus-shaped pattern that can be flexibly rotated around an axis. In this study, the antenna is investigated only in two principal modes: Common (C), where the antenna is fed with $0^{\circ}$ phase shift between its two ports and therefore produces omnidirectional $\mathrm{CP}$ in the horizontal plane (i.e. $x z$-plane for antenna 1 or $x y$ - 
plane for antenna 2); and Differential (D) where the antenna is fed with $180^{\circ}$ phase shift between its two ports and therefore produces omnidirectional $\mathrm{CP}$ in the elevation plane (i.e. $y z$-plane for both antennas). It should be noted, that this two modes does not fully exploit the antenna's capabilities, which allow continuous rotation of the pattern.

For CP the proper phase alignment, which steers the positive or negative interference at the pattern intersection points, can be easily realized by a phase shift between the two antennas, which also compensates for antenna rotation. This is given in Table I.

TABLE I. PhaSes OF ANTENNA FEEDS.

\begin{tabular}{|c|c|c|c|c|c|c|c|}
\hline \multirow{2}{*}{$\begin{array}{c}\text { Beam } \\
\text { dir. }\end{array}$} & \multicolumn{3}{|c|}{ Mode } & \multirow{2}{*}{\begin{tabular}{c} 
Shift \\
\cline { 5 - 8 }
\end{tabular}} & Ant 1 & Ant 2 & \multicolumn{4}{|c|}{ A1-A2 } & P1 & P2 & P3 & P4 \\
\hline$-\mathrm{x}$ & $\mathrm{C}$ & $\mathrm{C}$ & $-90^{\circ}$ & $0^{\circ}$ & $0^{\circ}$ & $270^{\circ}$ & $270^{\circ}$ \\
\hline$+\mathrm{x}$ & $\mathrm{C}$ & $\mathrm{C}$ & $+90^{\circ}$ & $0^{\circ}$ & $0^{\circ}$ & $90^{\circ}$ & $90^{\circ}$ \\
\hline$-\mathrm{y}$ & $\mathrm{D}$ & $\mathrm{C}$ & $180^{\circ}$ & $180^{\circ}$ & $0^{\circ}$ & $0^{\circ}$ & $0^{\circ}$ \\
\hline$+\mathrm{y}$ & $\mathrm{D}$ & $\mathrm{C}$ & $0^{\circ}$ & $0^{\circ}$ & $180^{\circ}$ & $0^{\circ}$ & $0^{\circ}$ \\
\hline$-\mathrm{z}$ & $\mathrm{C}$ & $\mathrm{D}$ & $0^{\circ}$ & $0^{\circ}$ & $0^{\circ}$ & $0^{\circ}$ & $180^{\circ}$ \\
\hline$+\mathrm{z}$ & $\mathrm{C}$ & $\mathrm{D}$ & $180^{\circ}$ & $0^{\circ}$ & $0^{\circ}$ & $180^{\circ}$ & $0^{\circ}$ \\
\hline
\end{tabular}

III. SiMULATED RESULTS

Fig. 2 shows the simulated results (CST Microwave Studio) for realized gains when the antenna is fed with a combination of phases required for $\pm x$ and $\pm z$ beams in the $x z$-plane (as seen in Table I). Results for $\pm y$ beams are similar to those of $\pm z$ and therefore not shown for brevity. The maximum realized gain varies from $3.1 \mathrm{dBic}$ for $+z$ beam to $2 \mathrm{dBic}$ for $\pm x$ beams. It is less than theoretical prediction in [2] and is due to the $\lambda / 2$ spacing between antennas. It can be seen, that the sidelobes are produced in the hemisphere opposite to the main beam, which is due to the same reason. Upon placing the antennas closer to each other, the sidelobes will vanish and the directivity (and gain) will therefore increase. It can be seen, that both sidelobes and decreased gain are more pronounced for the $\pm x$ configurations, i.e. where the main beam is produced along the same axis as the physical separation between antennas.

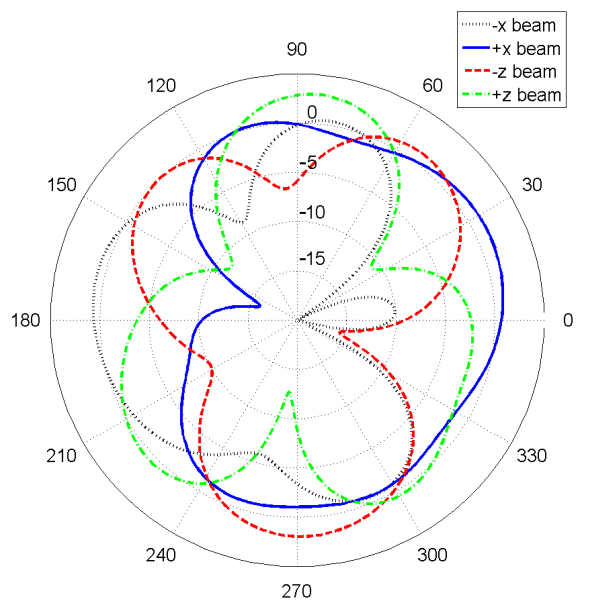

Fig. 2. RHCP realized gains (dBic) in the xz-plane at 2.46
$\mathrm{GHz}$ for various feed configurations. Axis $+x$ corresponds to angle $\theta=0^{\circ}$ and axis $+z-$ to angle $\theta=90^{\circ}$.

Fig. 3 shows the axial ratios (AR) for the same configuration. The $3 \mathrm{~dB}$ AR coverage is asymmetric between the two principal cuts: i.e. the $+x$ beam produce a wide AR beamwidth in the $x z$-plane (as seen in Fig. 3), but narrower in the $x y$-plane. On the contrary, the $-x$ beamwidth is narrow in $x z$-plane (seen in Fig. 3) and wide in $x y$-plane. This is considered a likely side effect of the antenna orientation.

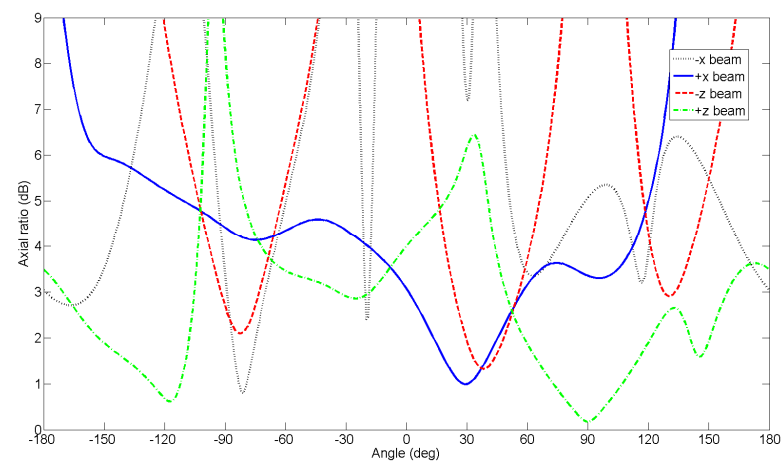

Fig. 3. Axial ratios in the $x z$-plane at $2.46 \mathrm{GHz}$.

\section{CONCLUSION}

The paper demonstrates how a simple array of two reconfigurable antennas can be combined into a Huygens source. This is used to produce 6 radiation patterns, each radiating in a different direction. This performance offers benefits for MIMO or pattern steering applications. As the steering is realized only by a phase shift, each channel can be steered independently.

Future work will focus on miniaturization of the antenna to allow a smaller separation between antennas and therefore further enhance the gain and minimize sidelobes.

\section{ACKNOWLEDGEMENT}

This work was supported by "ELEVATE: Irish Research Council International Career Development Fellowship - cofunded by Marie Cure Actions", grant number: ELEVATEPD/2014/79.

\section{REFERENCES}

[1] A. Narbudowicz, X. Bao, M. Ammann, H. Shakhtour, and D. Heberling, "Circularly Polarized Antenna With Steerable Dipole-Like Radiation Pattern," IEEE Trans. On Antennas and Propagation, vol. 62, issue 2, pp. 519-526, February 2013.

[2] S. R. Best, "Progress in the Design and Realization of an Electrically Small Huygens Source," In Proc.: 2010 International Workshop on Antenna Technology (iWAT), Lisbon, Portugal, 1-3 March 2010.

[3] P. Alitalo, A. O. Karilainen, T. Niemi, C. R. Simovski, and S. A. Tretyakov, "Design and realisation of an electrically small Huygens source for circular polarisation," IET Microwaves, Antennas \& Propagation, vol. 5, issue 7, pp. 783-789, May 2011.

[4] A. O. Karilainen, and S. A. Tretyakov, "Circularly Polarized Receiving Antenna Incorporating Two Helices to Achieve Low Backscattering," IEEE Trans. On Antennas and Propagation, vol. 60 , issue 7, pp. 3471-3475, July 2012. 